Pacific Journal of Mathematic 


\section{SOME PROPERTIES OF DISTRIBUTIONS \\ ON LIE GROUPS}

\section{LEON EHRENPREIS}

1. Introduction. Let $G$ be a separable Lie group and let $V$ be a complete, metrizable, topological vector space. The underlying space of $G$ is a separable real analytic manifold so that we can define, by the methods of L. Schwartz (see [7], [12], [13]), the spaces $\mathscr{E}(V)$ of indefinitely differentiable maps of $G$ into $V$, and $\mathscr{D}(V)$ which consists of those maps in $\mathscr{E}(V)$ which are of compact carrier. Their duals are $\mathscr{D}^{\prime}(V)$, the space of distributions on $G$ with values in $V^{\prime}$ (the dual of $V)$, and $\mathscr{E}^{\prime}(V)$ which is the space of distributions of compact carrier with values in $V^{\prime}$.

By using the group structure in $G$, we can define the convolution $S * f \in \mathscr{E}(C)$ for any $S \in \mathscr{D}^{\prime}(V), f \in \mathscr{D}(V)$, where $C$ is the complex plane. The main result of this paper is: Let $S \in \mathscr{D}^{\prime}(V)$ have the property that $S * f \in \mathscr{D}(C)$ whenever $f \in \mathscr{D}(V)$; then $S \in \mathscr{E}^{\prime}(V)$. Moreover, the topology of $\mathscr{E}^{\prime}(V)$ is that obtained by considering each $S \in \mathscr{E}^{\prime}(V)$ as defining the continuous linear transformation $f \rightarrow S * f$ of $\mathscr{D}(V) \rightarrow$ $\mathscr{D}(C)$ and then giving this set of transformations the compact-open topology (see [6]). This generalizes the result of [6] in case $G$ is a vector group and $V=C$.

This result is generalized to double coset spaces $L \backslash G / K$ where $L$ and $K$ are compact subgroups of $G$. In this form, the result will be used by the author and F.I. Mautner to generalize the Paley-Wiener theorem and the theory of mean-periodic functions of Schwartz (see $[8])$.

The author wishes to express his thanks to Professor F. I. Mautner for helpful discussions.

2. Distributions on G. Instead of using the usual method of defining distributions on $G$, as for example in de Rham and Kodaira [12], we shall follow another approach which is more akin to the author's thesis [5]. We shall show that the two methods are equivalent.

By "function" we shall mean "complex-valued function" unless the contrary is specifically stated. "Linear" will mean "linear over the complex numbers" always. By 1 we denote the identity in $G$, and by $g$ we denote the Lie algebra of $G$. For any $Y \in \mathfrak{g}$, we denote by $t \rightarrow \exp (t Y)$ the unique one parameter subgroup in $G$ whose direction

Received September 22, 1955. Work partially supported by National Science Foundation Grant NSF5-G1010. 
at 1 is $Y$. Let $V$ be a complete metrizable locally convex topological vector space.

The map $f$ of $G$ into $V$ is said to be differentiable in the direction $Y \in \mathfrak{g}$ at $x \in G$ if $\left\{\left(\frac{d f}{d t}\right)[(\exp t Y) x]\right\}_{t=0}$ exists ; if this is the case, we set

$$
\left(D_{Y} f\right)(x)=\left\{\left(\frac{d f}{d t}\right)[(\exp t Y) x]\right\}_{t=0} .
$$

If $f$ is a continuous map of $G$ into $V$, we say that $f$ is in the domain of $D_{Y}$ if, for any $x \in G, f$ is continuously differentiable in the direction $Y$ at $x . \quad D_{Y} f$ is then defined as the (continuous) map $x \rightarrow\left(D_{Y} f\right)(x)$.

By $\mathscr{E}^{0}$ we denote the space of continuous maps of $G$ into $V$ with the topology of uniform convergence in $V$ on the compact sets of $G$. By the carrier of an $f \in \mathscr{E}^{0}$ we mean the closure of the set of points where $f \neq 0$. An operator on $G$ is a linear mapping of a subspace of $\mathscr{E}^{0}$ into $\mathscr{E}^{\circ}$. The operator $D$ is said to be closed if the conditions: $\left\{f_{a}\right\}$ in the domain of $D, f_{\alpha} \rightarrow f$ and $D f_{\alpha} \rightarrow h$ in $\mathscr{E}^{0}$, imply $f$ is in the domain of $D$ and $D f=H$.

Proposition 1. For any $Y$ in $\mathfrak{g}, D_{Y}$ is a closed operator.

Proof. It is clear that $D_{Y}$ is an operator.

It remains to show that $D_{Y}$ is closed. Let $\left\{f_{i}\right\}$ be a sequence of functions in the domain of $D_{Y}$ such that $\left\{f_{i}\right\}$ and $\left\{D_{Y} f_{i}\right\}$ are Cauchy sequences in $\mathscr{E}^{0}$; call $f=\lim f_{i}, h=\lim D_{Y} f_{i}$, the limits being taken in $\mathscr{E}^{\circ}$. Let $Y, X_{2}, X_{3}, \cdots, X_{n}$ be a basis for $g$ and $N$ an open neighborhood of 1 in $G$ in which $\exp \left(t_{1} Y\right) \exp \left(t_{2} X_{2}\right) \cdots \exp \left(t_{n} X_{n}\right)$ form a coordinate system. It is clearly sufficient to prove that $f$ is in the domain of $D_{Y}$ at 1 and that $\left(D_{Y} f\right)(x)=h(x)$ for any $x \in N$.

Now, $\theta:\left(t_{1}, t_{2}, \cdots, t_{n}\right) \rightarrow\left(\exp \left(t_{1} Y\right), \exp \left(t_{2} X_{2}\right), \cdots, \exp \left(t_{n} X_{n}\right)\right)$ maps a circular neighborhood $M$ of 0 in real Euclidean $n$-space homeomorphically onto $N$. It is immediate from the definitions that a continuous map $p$ of $G$ into $V$ is differentiable in the direction $Y$ at 1 if and only if $p \theta$ has a continuous partial derivative in the direction $t_{1}$ at 0 , and then

$$
\left(D_{Y} p\right)(x)=\left(\frac{\partial p \theta}{\partial t_{1}}\right)(x)
$$

for all $x$ in a suitable neighborhood of 1 . From this and the known closure of $\partial / \partial t_{1}$ on Euclidean space, our assertion follows.

Now, let $Y_{1}, Y_{2}, \cdots, Y_{n}$ be a basis for $\mathfrak{g}$. We set

$$
D_{1}=D_{Y_{1}}, D_{2}=D_{Y_{2}}, \cdots, D_{n}=D_{Y_{n}}
$$


and we call $\mathfrak{D}$ the family $\left(D_{1}, D_{2}, \cdots, D_{n}\right)$ so $\mathfrak{D}$ is a family of closed operators. By means of $D$ we can now define, by the methods of [5], the complete, locally convex, Hausdorff, topological vector spaces $\mathscr{D}$ (or $\mathscr{D}(V)$ ) of indefinitely differentiable maps of compact carrier of $G$ into $V$, and $\mathscr{E}$ (or $\mathscr{E}(V)$ ) of all indefinitely differentiable maps of $G$ into $V . \mathscr{E}$ is a metrizable space; a sequence $\left\{f_{i}\right\}$ converges to zero in $\mathscr{E}$ if and only if for any operator $D^{*}=D_{j_{1}} D_{j_{2}} \cdots D_{j_{r}}, D_{j_{m}} \in \mathfrak{D}, D f$ $\rightarrow 0$ uniformly in $V$ on every compact set of $G$. The topology of $\mathscr{D}$ may be described as follows: For each compact set $K$, let $\mathscr{D}_{K}$ be the subspace of $\mathscr{D}$ consisting of those maps of $\mathscr{D}$ which have their carriers in $K$; the topology of $\mathscr{D}_{K}$ is that induced by $\mathscr{E}$. Then of all possible locally convex topologies which induce on each $\mathscr{D}_{K}$ the topology of $\mathscr{D}_{K}$ that may be given to the set of functions of $\mathscr{D}, \mathscr{D}$ is given the strongest (see [4]).

Proposition 2. The spaces $\mathscr{D}$ and $\mathscr{E}$ are the same as those we would have obtained by considering $G$ as an indefinitely differentiable manifold. ${ }^{1}$

Proof. Let $N$ be a neighborhood of 1 in $G$ in which $\left(\exp t_{1} Y_{1}\right.$ $\left.\exp t_{2} Y_{2} \cdots \exp t_{n} Y_{n}\right)$ form a coordinate system. Then it is clearly sufficient to prove the theorem for the restrictions of the functions of $\mathscr{E}$ and $\mathscr{D}$ to $N$. The result now follows by the method of the proof of Proposition 1.

\section{Proposition 3. $\mathscr{D}$ and $\mathscr{O}$ are reflexive topological spaces. ${ }^{2}$}

Proof. We prove the theorem first for $\mathscr{E}$. Since $\mathscr{E}$ is metrizable, it is sufficient to prove that $\mathscr{E}$ is a Montel space, that is, that the bounded sets of $\mathscr{E}$ are relatively compact (of compact closure). Let then $B$ be a bounded set in $\mathcal{E}$. Let $N$ be a compact neighborhood of 1 in $G$ in which $\left(\exp t_{1} Y_{1} \exp t_{2} Y_{2} \cdots \exp t_{n} Y_{n}\right)$ form a coordinate system. Since $G$ is separable, we can find a sequence of points $a_{i} \in G$ such that $G=\bigcup\left(\right.$ interior $\left.N a_{i}\right)$.

It is easily seen that it is sufficient to show that, for any $i$, and for any integers $r_{1}, r_{2}, \cdots, r_{m}$, if we set $D^{*}=D_{r_{1}} D_{r_{2}} \cdots D_{r_{m}}$, then the set $\left\{D^{*} f\right\}_{f \in B}$ is equicontinuous on $a_{i} N$. It follows immediately as in the proof of Proposition 1 that the restrictions of the maps $D^{*} f$ have the property that (if we identify them with maps on a circular neighborhood of zero in Euclidean $n$-space) their partial derivatives in all direc-

1 That is, by applying the method of de Rham and Kodaira [12].

2 See [3]. 
tions are uniformly bounded for $f \in B$. As is well-known, this implies the equicontinuity of $\left\{D^{*} f\right\}_{f \in B}$ on $a_{i} N$; hence Proposition 3 is established as regards the space $\mathscr{E}$.

If $L$ is a bounded set in $\mathscr{D}$, then all the maps of $L$ have their carriers in a fixed compact set $K$ of $G$, that is, $L \subset \mathscr{D}_{K}$. Since the topology induced by $\mathscr{D}$ on $\mathscr{D}_{K}$ is also the topology induced by $\mathscr{E}$ on $\mathscr{D}_{K}, L$ is bounded in $\mathscr{E}$. Thus, $L$ is relatively compact in $\mathscr{E}$, hence in $\mathscr{D}_{K}$, hence also in $\mathscr{D}$ which concludes the proof of Proposition 3.

A sequence of open, relatively compact (that is, of compact closure) sets $K_{i} \subset G$ will be called a scattered resolution of $G$ (see [5]) if $\cup K_{i}$ $=G$ and if, given any compact set $K \subset G$, only a finite number of the $K_{i}$ meet $K$. Given any scattered resolution $\left\{K_{i}\right\}$ of $G$, there exists a partition of unity $\left\{h_{i}\right\}$ relative to it; by this is meant that the indefinitely differentiable functions $h_{i}$ have the properties that:

\section{For each $i$, carrier $h_{i} \subset K_{i}$.}

2. For any $x \in G, \Sigma h_{i}(x)=1$.

(This sum has meaning because all but a finite number of terms are zero.) To establish the existence of the partition of unity $\left\{h_{i}\right\}$, we have only to note that the scattered resolution $\left\{K_{i}\right\}$ can be "refined" to a scattered resolution $\left\{L_{i}\right\}$ by coordinate neighborhoods (that is, each $K_{i}$ is contained in a union of a finite number of $L_{j}$ ). The existence of a partition of unity relative to $\left\{L_{i}\right\}$ is readily verified and, in turn, implies immediately the existence of a partition of unity relative to $\left\{K_{i}\right\}$.

By $\mathscr{D}^{\prime}$ (or $\mathscr{D}^{\prime}(V)$ we denote the dual of $\mathscr{D}$ with the topology of uniform convergence on the bounded (compact) sets of $\mathscr{D}$. It can be shown (see [7]) that, $\mathscr{D}^{\prime}$ can also be described as the space of continuous linear maps of $\mathscr{D}(C) \rightarrow V^{\prime}$, this space of maps being given the compact-open topology. For this reason, $\mathscr{D}^{\prime}$ is usually called the space of distributions on $G$ with values in $V^{\prime}$. In this paper, we shall call the elements of $\mathscr{D}^{\prime}$ distributions.

For any distribution $S$, and any open set $O$ in $G$, we say that $S$ vanishes on $O$ if $S \cdot f=0$ for any $f \in \mathscr{D}$ whose carrier is contained in $O$. Because of the existence of partitions of unity, we can easily show that if $S$ vanishes on $O_{a}$ where $O_{a}$ are open sets, then $S$ vanishes also on $\cup O_{\alpha}$. Thus there is a largest open set on which $S$ vanishes. The carrier of $S$ is defined as the complement of this set.

$\mathscr{E}^{\prime}$ (or $\mathscr{E}^{\prime}(V)$ ) is the dual of $\mathscr{E}$. It can be shown, as in [13], that $\mathscr{E}^{\prime}$ consists of all distributions of compact carrier.

For any $S \in \mathscr{D}^{\prime}$, by $\bar{S}$ is meant the distribution $f \rightarrow \overline{S \cdot \bar{f}}$ for $f \in \mathscr{D}$, 
where $\overline{f(x)}=\overline{f(x)}$ for any $x \in G$.

By $G \times G$ we denote the direct product of $G$ with itself; $G \times G$ is again a Lie group whose underlying manifold is the Cartesian product of the underlying manifold of $G$ with itself. By ${ }_{2} \mathscr{Z},{ }_{2} \mathscr{E}, \mathscr{C}_{2},{ }_{2} \mathscr{C}^{\prime}$ we denote the spaces on $G \times G$ corresponding to $\mathscr{D}, \mathscr{E}, \mathscr{D}^{\prime} \mathscr{E}^{\prime}$ respectively.

Let $k$ be a continuous map on $G \times G$ and $x \in G$. Then by $k_{X_{1}=x}$ we mean the map on $G: y \rightarrow k(x, y)$. Suppose that, for all $x \in G, k_{x_{1}=x}$ is in a space $U$ of mappings on $G$. Then by $k_{1}$ we mean the mapping $x \rightarrow k_{X_{1}=x}$ of $G \rightarrow U$. Let $L$ be a map defined on $U$; then we say that $k$ is in the domain of $L$, and we denote by $L_{2} k$ the map

$$
x \rightarrow L k_{X_{1}=x}
$$

for $x \in G$. If the range of $L$ is again a space of mappings on $\mathrm{G}$, then we say also that $k$ is in the domain of $L_{21}$ and we shall denote by $L_{21} k$ the mapping on $G \times G$ :

$$
(x, y) \rightarrow L k_{X_{1}=x}(y) .
$$

$L_{21}$ is called the lift of $L$ to $G \times G$. We define $k_{X_{2}=x}, k_{2}, L_{1}, L_{1 /}$ similarly.

We can now define, as in [5], two products involving distributions and functions:

For any $S \in \mathscr{D}^{\prime}, k \in, \mathscr{D}$, then we have two inner products: $S_{1} k$ and $S_{2} k$ which are both in $\mathscr{D}$.

For any $S, U \in \mathscr{D}^{\prime}$ we define the direct products $S_{1} \times U_{2}$ and $S_{2} \times U_{1}$ $\epsilon_{2} \mathscr{D}^{\prime}$ by

$$
S_{1} \times U_{2} \cdot k=S \cdot U_{2} k, \quad S_{2} \times U_{1} \cdot k=S \cdot U_{1} k
$$

for any $k \in_{2} \mathscr{D}$.

The direct products define continuous bilinear maps which are commutative, while the inner products are only separately continuous bilinear maps. (If $V, W, X$ are topological vector spaces and $t: V \times W \rightarrow$ $X$ is a bilinear map, then $t$ is called separately continuous (see [4], [5]) if, for $B, B^{\prime}$ any bounded sets in $V, W$ respectively, the maps

$$
w \rightarrow t(b, w), v \rightarrow t\left(v, b^{\prime}\right)
$$

are, for $b \in B, b^{\prime} \in B^{\prime}$, equicontinuous linear maps of $W \rightarrow X$ and $V \rightarrow X$ respectively.)

By $\left\{Q_{i}\right\}$ we shall denote an enumeration of the operators $D_{r_{1}} D_{r_{2}} \cdots$ $D_{r_{m}}$ with $Q_{1}=$ identity.

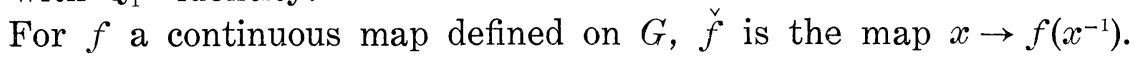

We shall denote by $\eta$ the function on $G$ defined by $d x g=\eta(g) d x$, where $d x$ is a left invariant Haar measure. It is known that $\eta \in \mathscr{E}(C)$ 
and, moreover, $\eta$ is a homomorphism on $G$. By $\omega$ we denote the function on $G$ defined by $d x^{-1}=\omega(x) d x$. Again, $\omega \in \mathscr{E}(C)$ and $\omega$ is a homomorphism on $G$. It is readily verified that $\omega(y)=\eta\left(y^{-1}\right)$ for any $y \in G$. For any $S \in \mathscr{D}^{\prime}$, we write $\check{S} \cdot f=S \cdot \omega \check{f}$ for any $f \in \mathscr{D}$.

3. Convolution on G. For any continuous map $f$ of $G$ into $V$ and any $x \in G$ we define the translations

$$
(\mathfrak{Q}(x) f)(y)=f\left(x^{-1} y\right) \quad(\Re(x) f)(y)=f(y x)
$$

for any $y \in G$.

Proposition 4. $(x, f) \rightarrow \mathfrak{L}(x) f$ and $(x, f) \rightarrow \mathfrak{R}(x) f$ are continuous maps of $G \times \mathscr{D} \rightarrow \mathscr{D}$ and also of $G \times \mathscr{E} \rightarrow \mathscr{E}$.

Proof. We shall establish the theorem for the map $(x, f) \rightarrow \mathfrak{R}(x) f$ of $G \times \mathscr{D} \rightarrow \mathscr{D}$; the other parts of the proposition may be established by similar methods. By the results of Dieudonné and Schwartz (see [4], [5]) it is sufficient to prove that this is a continuous map of $G \times \mathscr{D}_{K}$ $\rightarrow \mathscr{D}$ for any compact set $K$ of $G$. Since the map is linear in $f$ and a homomorphism in $x$, it is sufficient to prove continuity at $f=0$ and $x=1$. Let $K$ be a given compact set in $G$ and choose $K^{\prime}$ a compact set in $G$ so large that $K^{\prime}$ contains the carriers of all $\mathscr{L}(x) f$ for $x \in \mathscr{D}_{K}$. Let $M$ be a neighborhood of zero in $\mathscr{D}_{K^{\prime}}$. Then we can find operators $Q_{1}, Q_{2}, \cdots, Q_{r}$, and continuous semi-norms $\rho_{1} . \rho_{2}, \cdots, \rho_{n}$ on $V$, and a positive number $a$ so that $M$ contains the set of $h \in \mathscr{D}_{K^{\prime}}$ which satisfy

$$
\max _{y \in G, i} \rho_{i}\left[\left(Q_{j} h\right)(y)\right] \leqq a
$$

for $j=1,2, \cdots, r$.

For any $p \in \mathscr{D}$, any $k$, and $x, z \in G$,

$$
\begin{aligned}
\left(D_{k} \mathcal{L}(z) p\right)(x) & =\left\{\left[\left(\frac{d}{d t}\right) \mathfrak{R}(z) p\right]\left[\left(\exp t Y_{k}\right) x\right]\right\}_{t=0} \\
& =\left\{\left[\left(\frac{d}{d t}\right) p\right]\left[z^{-1}\left(\exp t Y_{k}\right) x\right]\right\}_{t=0} \\
& =\left\{\left(\frac{d p}{d t}\right)\left[z^{-1}\left(\exp t Y_{k}\right) z z^{-1} x\right]\right\}_{t=0} \\
& =\left\{\left(\frac{d p}{d t}\right)\left[\left(\exp t z^{-1} Y_{k} z\right) z^{-1} x\right]\right\}_{t=0} .
\end{aligned}
$$

Now, write $z^{-1} Y_{k} z=\Sigma c_{k l}(z) Y_{l}$ where $\left(c_{k l}\right)$ is the matrix of the adjoint representation of $G$ on $g$. Then we have 


$$
\left(D_{k} \mathfrak{Q}(z) p\right)(x)=\left(D_{z^{-1} Y_{k}} f\right)\left(z^{-1} x\right)
$$

We also have

$$
D_{z}^{-{ }^{-1} Y z} p=\Sigma c_{k j}(z) D_{j} p .
$$

The functions $c_{k j}$ are continuous and even indefinitely differentiable on $G$. Hence, we can find an $A>0$ so that

$$
\max _{z \in K}\left|c_{k j}(z)\right| \leqq A
$$

for all $k, j$.

It follows immediately from this that we can be assured that, for $q \in \mathscr{D}_{K}, z \in K$,

$$
\max _{x \in G, i} \rho_{i}\left[\left(D_{K} \mathfrak{Q}(z) q\right)(x)\right]
$$

will be small by making

$$
\max _{x \in G, j, i} \rho_{i}\left[\left(D_{j} q\right)(x)\right]
$$

sufficiently small. Proposition 4 now follows by iteration, since each $Q_{l}$ is of the form $D_{r_{1}} D_{r_{2}} \cdots D_{r_{m}}$.

For any continuous map $f$ on $G, \& f$ is the map on $G \times G:(x, y) \rightarrow$ $f\left(x^{-1} y\right) ; \mathbb{2}^{*} f$ is the map on $G \times G:(x, y) \rightarrow f(x y)$. By the method of proof of Proposition 1, we can establish

Proposition 5. $f \rightarrow \mathfrak{Q} f$ and $f \rightarrow \mathfrak{2}^{*} f$ are continuous linear maps of $\mathscr{E} \rightarrow_{2} \mathscr{E}$.

We are now in a position to define the convolution product involving distributions and functions. The definition differs slightly from that of Schwartz [13]: For any $S \in \mathscr{D}^{\prime}, f \in \mathscr{D}, x \in G$, we set

$$
(S * f)(x)=\bar{S} \cdot 2(x) \check{f}
$$

This formula can also be considered valid if $S \in \mathscr{E}^{\prime}$ and $f \in \mathscr{E}$.

Proposition 6. $(S, f) \rightarrow S * f$ is a separately continuous map of
(a) $\mathscr{E}^{\prime} \times \mathscr{E} \rightarrow \mathscr{E}(C)$
(b) $\quad \mathscr{E}^{\prime} \times \mathscr{D} \rightarrow \mathscr{D}(C)$
(c) $\mathscr{D}^{\prime} \times \mathscr{D} \rightarrow \mathscr{E}(C)$.

which is antilinear in $S$ and linear in $f$. 
Proof. (a) Let $j$ be fixed and write $A=D_{j}$. We find from the definitions that, for $S \in \mathcal{E}^{\prime}, f \in \mathcal{E}, S * f \in \mathcal{E}(C)$ and, moreover,

$$
[A(S * f)](x)=\bar{S} \cdot\left(A_{1 /} \& \stackrel{\sim}{f}\right)_{X_{1}=x} .
$$

From this it follows by iteration that, for any $Q=Q_{s}$, we have

$$
[Q(S * f)](x)=\bar{S} \cdot\left(Q_{1 /} R \check{f}\right)_{X_{1}=x} .
$$

Part (a) results immediately from (3) together with Proposition 5.

(b) By a result of Dieudonné and Schwartz (see [4]) it is sufficient to prove that, for $K$ a compact set in $G,(S, f) \rightarrow S * f$ is a separately continuous map of $\mathscr{E}^{\prime} \times \mathscr{D}_{K} \rightarrow \mathscr{B}$. Now, it is obvious that

$$
\text { (carrier } S * f) \subset(\text { carrier } S)(\text { carrier } f) \text {. }
$$

Our assertion now follows from (a) above and the fact that $\mathscr{Z}_{K}$ has the topology induced by $\mathscr{E}$.

(c) This is proven by essentially the same reasoning as that employed in the proof of (a) above.

4. $\mathscr{E}$ as a space of linear transformations. In this section we shall prove our main result.

THEOREM 1. Let $S \in \mathscr{D}^{\prime}$ have the property that $S * f \in \mathscr{D}(C)$ whenever $f \in \mathscr{D}$; then $S \in \mathscr{E}^{\prime}$.

Proof. Let us suppose that $S$ satisfies the hypotheses of Theorem 1 , and let $K$ be a fixed compact set in $G$. We shall show first that there exists a compact set $K^{\prime} \subset G$ such that $S * \mathscr{D}_{K} \subset \mathscr{D}_{K^{\prime}}$. Assume this is not the case, and let $\left\{K_{i}\right\}$ be a compact exhaustion of $G$. (That is, each $K_{i}$ is a compact set which is the closure of a nonempty open set. Moreover, $K_{i} \subset K_{i+1}$ and $\cup K_{i}=G$.) We shall produce a sequence $\left\{g_{i}\right\}$ with the following properties:

1. Each $g_{i} \in \mathscr{D}_{K}$.

2. $\Sigma g_{i}$ converges in $\mathscr{D}_{K}$.

3. There is a sequence of positive numbers $m_{i}$ with $m_{i+1}-m_{i} \geqq 1$ for all $i$ such that

$$
\begin{aligned}
& \text { carrier }\left(S * g_{i}\right) \subset K_{m_{i}} \\
& \text { carrier }\left(S * g_{i+1}\right) \not \subset K_{m_{i}} .
\end{aligned}
$$

4. There is a sequence of points $a_{i} \in G$ such that $a_{i}$ is a point of $K_{m_{i}}-K_{m_{i-1}}$ (where $K_{m_{0}}$ is the empty set) for which $\left(S * g_{i}\right)\left(a_{i}\right) \neq 0$ and 


$$
\left|\left(S * g_{i+k}\right)\left(a_{i}\right)\right| \leqq \frac{1}{3^{k}}\left|\left(S * g_{i}\right)\left(a_{i}\right)\right|
$$

for all $k>0$.

Suppose that the sequences $\left\{g_{i}\right\},\left\{m_{i}\right\},\left\{a_{i}\right\}$ can be found. Then for any $i>1$,

$$
\begin{aligned}
\left|\left(S * \Sigma g_{j}\right)\left(a_{i}\right)\right| & =\left|\Sigma\left(S * g_{j}\right)\left(a_{i}\right)\right| \\
& =\left|\Sigma_{j \geq i}\left(S * g_{j}\right)\left(a_{i}\right)\right| \\
& \geq\left|\left(S * g_{j}\right)\left(a_{i}\right)\right|-\sum_{j>i}\left|\left(S * g_{j}\right)\left(a_{i}\right)\right| \\
& \geq\left|\left(S * g_{i}\right)\left(a_{i}\right)\right|\left[1-\sum_{j \geq 1} \frac{1}{3^{j}}\right] \\
& =\frac{1}{2}\left|\left(S * g_{i}\right)\left(a_{i}\right)\right| \\
& >0 .
\end{aligned}
$$

Since the set $\left\{a_{i}\right\}$ is clearly not contained in any compact set of $G$, we conclude that $S * \Sigma g_{j}$ is not of compact carrier, which contradicts our hypothesis.

It remains to define the sequences $\left\{g_{i}\right\},\left\{m_{i}\right\}$, and $\left\{a_{i}\right\}$. Let $g_{1} \in \mathscr{D}_{K}$ be chosen so that $S * g_{1} \neq 0$. Let $a_{1}$ be any point in $G$ for which $\left(S * g_{1}\right)\left(a_{1}\right) \neq 0$, and choose $m_{1}>0$ so that

$$
\text { carrier }\left(S * g_{1}\right) \subset K_{m_{1}} \text {. }
$$

Assume that $g_{1}, \cdots, g_{k}, a_{1}, \cdots, a_{k}, m_{1}, \cdots, m_{k}$ have been defined with the required properties; we shall now define $g_{k+1}, a_{k+1}, m_{k+1}$. Now, by our assumption, there is an $f \in \mathscr{D}_{K}$ such that

$$
\text { carrier }(S * f) \not \subset K_{m_{k+1}} \text {. }
$$

Let $m_{k+1}$ be chosen so that carrier $(S * f) \subset K_{m_{k+1}}$, and let $a_{k+1}$ be some point in $K_{m_{k+1}}-K_{m_{k}}$ such that $(S * f)\left(a_{k+1}\right) \neq 0$. Define

$$
g_{k+1}=\frac{f}{\max \left(1, \max _{x \in G}|f(x)| 3^{k+1}\right) \max _{j, i \leqq k}\left(1, \max _{x \in G}\left[\left(Q_{i} f\right)(x)\right],\left[\left|\left(S * g_{i}\right)\left(a_{i}\right)\right|\right]^{-1}\right)}
$$

The sequences $\left\{g_{i}\right\},\left\{m_{i}\right\},\left\{a_{i}\right\}$ are thus defined. It is clear that conditions 1, 3, 4 are satisfied. Further, each $g_{i} \in \mathscr{D}_{K}$ and, for $R$ any semi-norm on $D_{K}$ of the kind used to define the topology of that space, it is clear that

$$
\Sigma R\left(g_{1}\right)>\infty
$$

Thus $\Sigma g_{i}$ converges in $\mathscr{D}_{K}$. 
To complete the proof of Theorem 1, let us assume that $S$ is not of compact carrier, and let $K$ be a given compact symmetric neighborhood of 1 in $G$. It is clear that we can choose an open set $U$ in $G$ such that $S$ does not vanish on $U$ and such that $U \cap K^{\prime}$ is empty, where $K^{\prime}$ is a compact symmetric set such that $\bar{S} * \mathscr{D}_{K} \subset \mathscr{D}_{K^{\prime}}$. It follows easily that we can find a $g \in G$, and an $f \in D$ such that carrier $f \subset K g \subset U, S \cdot f \neq 0$.

On the other hand, by definition,

$$
S \cdot \stackrel{v}{f}=(\bar{S} * \mathbb{Q}(g) f)\left(g^{-1}\right) .
$$

But, carrier $f \subset K g$ implies carrier $\mathfrak{L}(g) f \subset K$ because $K$ is symmetric. Also, $g \notin K^{\prime}$ because $1 \in K$ and $K^{\prime} \cap U$ is empty. Since $K^{\prime}$ is symmetric, also $g^{-1} \notin K^{\prime}$. Thus, $S \cdot \check{f}=(\bar{S} * \mathfrak{L}(g) f)\left(g^{-1}\right)=0$; this contradiction completes the proof of Theorem 1 .

The set of distributions of $\mathscr{E}^{\prime}$ forms a vector space of continuous linear mappings of $\mathscr{D} \rightarrow \mathscr{D}$ under convolution; we give this space the compact-open topology (see [6]) and obtain a topological vector space $J$. A fundamental system of neighborhoods of zero in $J$ consists of all sets $N$ for which we can find a compact set $K$ in $\mathscr{D}$ and a neighborhood of zero $M$ in $\mathscr{D}$ so that $N$ consists of those $S \in \mathscr{E}^{\prime}$ with $S * h$ $\in M$ for all $h \in K$. By Proposition 1 of $\S 5$ of [6], we would have obtained the same topologies if we had considered the distributions of $\mathscr{E}^{\prime}$ as defining, under convolution, continuous linear maps of $\mathscr{D}^{\prime} \rightarrow \mathscr{D}^{\prime}$.

THEOREM 2. The natural map $u: \mathscr{E}^{\prime} \rightarrow J$ is a topological isomorphism onto.

Proof. $u$ is clearly one-to-one, linear, and onto. Moreover, $J$ is given the weakest topology to make the maps

$$
S \rightarrow\left(u^{-1} S\right) * f
$$

of $J \rightarrow \mathscr{D}$ equicontinuous for $f$ in any compact set of $\mathscr{D}$; by Proposition 6 this implies that $u$ is continuous.

Since $u^{-1}$ is linear, we need verify continuity only at zero. Let $T$ be a neighborhood of zero in $\mathscr{E}^{\prime}$; there is a bounded set $\beta \subset \mathscr{E}$ so that $T$ contains the set of $S \in \mathscr{E}^{\prime}$ which satisfy $|S \cdot b| \leqq 1$ for all of $b \in \beta$.

Let $K$ be an open symmetric neighborhood of 1 in $G$ whose closure is compact. Then it is clear that we can find a sequence of points $a_{i}$ $\in G$ such that $\left\{a_{i} K\right\}$ is a scattered resolution of $G$ (see $\S 2$ ). We can also insure that, if $a$ is one of the $a_{i}$, so is $a^{-1}$. Let $\{h\}$ be a partition of unity relative to this scattered resolution (see $\S 2$ ). It is readi- 
ly verified by the method of proof of Proposition 1 of $\S 3$ that, for each $i$, the set $B_{i}$ of functions $\mathcal{L}\left(a_{i}\right)\left(h_{i} f\right)$ for $f \in \beta$ is bounded in $\mathscr{D}$. For each $j$ there is a double sequence $s_{j}=M_{j i k}$ of positive numbers so that $B_{j}$ is contained in the bounded (in $\mathscr{D}$ ) set $L_{j}$ of all $g \in D$ whose carriers are contained in $K$ and which satisfy

$$
\max _{x \in G} \rho_{k}\left[\left(Q_{i} g\right)(x)\right] \leqq M_{j i k}
$$

for all $i$. From the denumerable number of double sequences $s_{j}$ we construct a double sequence $s=\left\{M_{i k}\right\}$ of positive numbers such that, for each $j, M_{j i k} \leqq M_{i k}$ for all but a finite number of $i, k$. Hence, for each $j$, we can find an $e_{\jmath}>0$ so that $e_{j} M_{j i k} \leqq M_{i k}$ for all $i$, $k$; we can clearly make $e_{j}=e_{l}$ if $a_{j}=a_{l}^{-1}$.

Let $A$ be the set of $f \in \mathscr{D}$ for which

1. carrier $f \subset K$

2. $\max _{x \in G} \rho_{k}\left[\left(Q_{i} f\right)(x)\right] \leqq M_{i k}$ for all $i, k$,

so $A$ is bounded in $\mathscr{D}$. Let $M$ be the neighborhood of zero in $\mathscr{D}$ consisting of those $h \in \mathscr{D}$ with

$$
\max _{x \in a_{j} K} \rho_{k} h(x) \leqq e_{j} d_{j}
$$

for all $j$, where $d_{j}$ are positive numbers which satisfy $\Sigma d_{j}=1$. Call $N$ the set of $S \in J$ with $S * f \in M$ for all $f \in A$, so $N$ is a neighborhood of zero in $J$; we claim that $u^{-1}(N) \subset T$.

Let us assume this is not the case; then we can find an $S \in N$ with $u^{-1} S \notin T$, that is, $S \in N$ but

$$
\left|u^{-1} S \cdot f\right|>1
$$

for some $f \in \beta$. Now, $u^{-1} S$ is of compact carrier; thus we can find an $r$ such that

$$
\sum_{k=1}^{r} h_{k}(x)=1
$$

for any $x \in$ carrier $\left(u^{-1} S\right)$. Hence

$$
\left|u^{-1} S \cdot f\right| \leqq\left|u^{-1} S \cdot h_{1} f\right|+\left|u^{-1} S \cdot h_{2} f\right|+\cdots+\left|u^{-1} S \cdot h_{r} f\right| .
$$

It is clear from the definitions that, for each $i$,

$$
e_{i} \mathfrak{Q}\left(a_{i}\right)\left(h_{i} f\right) \in A \text {. }
$$

Thus, 


$$
h_{i} f=\frac{1}{e_{i}} \mathfrak{Q}\left(a_{i}^{-1}\right) g
$$

for some $g \in A$, which gives, for $i=1,2, \cdots, r$,

$$
\begin{aligned}
\left|u^{-1} S \cdot h_{i} f\right| & =\frac{1}{e_{i}}\left|u^{-1} S \cdot \mathcal{L}\left(a_{i}^{-1}\right) g\right| \\
& \left.=\frac{1}{e_{i}} \mid \overline{\left(u^{-1} S\right.} * g\right)\left(a_{i}^{-1}\right) \mid \\
& \leqq \frac{1}{e_{i}} e_{j} d_{j},
\end{aligned}
$$

where $a_{J}=a_{i}^{-1}$, because $g \in A$ and $u^{-1} S \in N$. Now, since $e_{i}=e_{j}$, we have

$$
\left|u^{-1} S \cdot h_{i} f\right| \leqq d_{j} .
$$

Applying this to equation (5) we obtain

$$
\left|u^{-1} S \cdot f\right| \leqq d_{1^{\prime}}+d_{2^{\prime}}+\cdots+d_{r^{\prime}} \leqq 1
$$

(where we set $a_{j^{\prime}}=a_{j}^{-1}$ ). This contradiction proves the theorem.

5. Extension of the main result. We assumed in $\S \S 2,3,4$ that $V$ is metrizable. In case $V$ is not metrizable, then the spaces $\mathscr{B}$ and $\mathscr{D}$ can be defined as before, but $E$ is no longer metrizable, and $\mathscr{D}$ is not an $\mathscr{L} \mathscr{F}$ space in the sence of Dieueonné and Schwartz [4]. However, there is no difficulty in extending the definition and continuity properties of the convolution product to this case. Theorem 1 can be extended to this case, but the proof of Theorem 2 does not extend to the case of $V$ not metrizable. All that can be proven (and the proof is much simpler than the proof of Theorem 2 above) is that $u$ is continuous and that $u^{-1}$ is sequentially continuous and takes bounded sets into bounded sets. The continuity of $u^{-1}$ is an open question.

We assume in the following that $V$ is a complete, locally convex, Hausdorff, topological vector space. By $V^{*}$ we denote the space of continuous linear maps of $V$ into $V$ with the compact-open topology, so $V^{*}$ is again a complete, locally convex, Hausdorff, topological vector space.

Let $K$ and $L$ denote compact subgroups of $G$. By a representation of $K$ on $V$ we mean a continuous homomorphism $U$ of $K$ into $V^{*}$. Let $U$ and $W$ be representations of $V$ of $K$ and $L$ respectively. By ${ }_{U W} \mathscr{D}$ we denote the space of those $f \in \mathscr{D}\left(V^{*}\right)$ for which

$$
\mathfrak{L}\left(k^{-1}\right) \Re(l) f=U(k) f W(l)
$$

for any $k \in K, l \in L$. We give ${ }_{U W} \mathscr{D}$ the topology induced by $\mathscr{D}$. WW $\mathscr{E}$ 
is defined similarly.

For any $T \in \mathscr{D}^{\prime}\left(V^{*}\right), g \in G$, we define $\mathcal{L}(g) T$ and $\Re(g) T$ as the distributions

$$
\mathfrak{Z}(g) T \cdot f=T \cdot \mathfrak{Q}\left(g^{-1}\right) f, \quad \Re(g) T \cdot f=\eta(g) T \cdot \Re\left(g^{-1}\right) f
$$

for any $f \in \mathscr{D}\left(V^{*}\right)$. ( $\eta$ was defined in $\S 2$.) Let us denote by ${ }_{U W} \mathscr{D}^{\prime}$ the space of all $S \in \mathscr{D}^{\prime}\left(V^{*}\right)$ which satisfy

$$
\mathfrak{2}(k) \Re(l) S \cdot f=S \cdot U\left(k^{-1}\right) f W(l)
$$

for any $f \in \mathscr{D}\left(V^{*}\right), k \in K, l \in L .^{3} \quad$ We shall write $U(k) S W\left(l^{-1}\right) \cdot f$ for the right side of (8). We give ${ }_{U W} \mathscr{D}^{\prime}$ the topology induced by $\mathscr{D}^{\prime}\left(V^{*}\right)$. ${ }_{U W} \mathscr{E}^{\prime}$ is defined similarly.

We can easily show

\section{Proposition 7.}

$$
f \rightarrow P_{U W} f=\int_{K \times L} U(k) \mathfrak{Q}(h) \Re(l) f V^{-1}(l) d k d l
$$

(where $d k$ and $d l$ are the respective Haar measures on $K$ and $L$ so normalized that $\left.\int_{K} d k=\int_{L} d l=1\right)$ defines continuous open projections of $\mathscr{D}\left(V^{*}\right)$ onto ${ }_{U W} \mathscr{D}$ and $\mathscr{E}\left(V^{*}\right)$ onto ${ }_{U W} \mathscr{E}$. Also

$$
S \rightarrow P_{U W} S=\int_{K \times L} \mathfrak{2}\left(k^{-1}\right) \Re(l)\left[U^{-1}(k) S V(l)\right] d k d l
$$

defines continuous open projections of $\mathscr{D}^{\prime}\left(V^{*}\right)$ onto ${ }_{w} \mathscr{D}^{\prime}$ and $\mathscr{E}^{\prime}\left(V^{*}\right)$ onto ${ }_{U W} \mathscr{E}^{\prime}$.

Corollary. ${ }_{W W} \mathscr{D}^{\prime}$ is the dual of ${ }_{W W} \mathscr{D}$ and ${ }_{U W} \mathscr{E}^{\prime}$ is the dual of UW $\mathscr{E}$.

Proof. This is an immediate consequence of Proposition 6 and the fact that, for $S \in \mathscr{D}^{\prime}, f \in \mathscr{D}$ (or for $S \in \mathscr{E}^{\prime}, f \in \mathscr{E}$ ), we have $P_{U W} S \cdot f$ $=S \cdot P_{U W} f$.

Suppose that $K=L$; then we see easily that the convolution defined in $\S 3$ defines a separately continuous bilinear map of ${ }_{U W} \mathscr{D}^{\prime} \times_{W Z} \mathscr{D} \rightarrow$ ${ }_{U Z} \mathscr{E}(C)$ (where $U, W, Z$ are representations of $K$ on $V$ ). The method of proof of Theorems 1 and 2 can be used to show.

THEOREM 3. UW $\mathscr{E}^{\prime}$ consists of all $S \in_{U W} \mathscr{D}^{\prime}$ such that $S * f \in_{U W} \mathscr{D}$ for any $f \in_{W W} \mathscr{D}$. The topolagy of ${ }_{W W} \mathscr{E}^{\prime}$ is sequentially the same as that obtained by considering the elements of ${ }_{U W} \mathscr{E}^{\prime}$ as defining (by convolution) continuous linear maps of ${ }_{W W} \mathscr{D} \rightarrow_{W W} \mathscr{D}$ and giving this set the

3 Note that since $L$ is compact, the restriction of $\eta$ to $L$ is 1 . 
compact-open topology $\tau$. Moreover, the bounded sets of ${ }_{U W} \mathscr{E}^{\prime}$ are the same as those of $\tau$.

REMARK 1. We do not know whether the topologies $\tau$ and that of ${ }_{U W} \mathcal{E}^{\prime}$ are the same. The difficulty is that, for $f \in_{W W} \mathscr{D}, g \in G, \mathfrak{L}(g) f$ is no longer in ${ }_{W W} \mathscr{D}$.

REMARK 2. In case that $K=L, V$ is finite dimensional, and $U, W$, $X, Z$ are irreducible unitary representations of $K$ on $V$, then it follows easily from the Schur orthogonality relations that $S * f=0$ for any $S \in$ ${ }_{U W} \mathscr{E}^{\prime} f \in_{X Z} \mathscr{D}$ if $W$ is not equivalent to $X$.

REMARK 3. The conclusion of Theorem 3 does not necessarily hold if the space ${ }_{W W} \mathscr{D}$ in the hypothesis of the theorem is replaced by ${ }_{W Z} \mathscr{D}$ where $Z$ is different from $W$, even if $V$ is finite dimensional and $U$, $W, Z$ are irreducible unitary representations of $K$. An example will be given in a forthcoming paper of the author and F. I. Mautner. ( $G$ can be taken as the complex unimodular group.)

6. General remarks. We have assumed that $G$ is a separable Lie group. In the general case, the spaces $\mathscr{E}$ and $\mathscr{D}$ can be defined as before, but $\mathscr{E}$ will not be metrizable and $\mathscr{D}$ will not be an $\mathscr{L} \mathscr{F}$ space in the sense of Dieudonné and Schwartz [4] because $\mathscr{D}$ will be the inductive limit of a non-denumerable number of spaces, For this reason, the topology of $\mathscr{D}$ is best defined as follows: Let $\left\{f_{i j}\right\}=\sigma$ be a family of continuous functions on $G$ such that

(a) For each $i$, only a finite number of $j$ appear.

(b) Only a finite number of $f_{i j}$ are different from zero on any compact set of $G$.

Then we define $N_{\sigma}$ as the set of $h \in \mathscr{D}$ for which

$$
\max _{x \in G} \rho_{j}\left[\left(f_{i j}(x) Q, h\right)(x)\right] \leqq 1
$$

for all $i, j$, where the $Q_{j}$ are as in $\S 2$, and $\left\{\rho_{i}\right\}$ denotes an enumeration of semi-norms which are sufficient to define the topology of $V$. The sets $N_{\sigma}$ are seen to form a fundamental system of neighborhoods of zero of a locally convex topological vector space which we shall call $\mathscr{D}$. In case $\mathscr{D}$ is separable it is easily verified that the two definitions agree.

The advantage of the above definition is that it implies immediately the completeness of $\mathscr{D}$. For, the completion of $\mathscr{D}$ obviously consists of indefinitely differentiable maps. Moreover, if $h$ is any map in the completion of $\mathscr{D}$, then, for any continuous function $f$ on $G$, and any 
$k$, it is easily seen that $\rho_{k}(f h)$ is a bounded function. This implies immediately that $h$ is of compact carrier, hence $h \in \mathscr{D}$.

The properties of convolution can be extended to the nonseparable case and there is no difficulty in extending part of our main results. We can, as in $\S 5$, prove only that the topology of $\mathscr{E}^{\prime}$ is sequentially, and in regard to bounded sets, the same as the compact-open topology of the space of linear transformations of $\mathscr{D} \rightarrow \mathscr{D}$ (under convolution).

The results of $\S 5$ on double coset spaces $K \backslash G / L$ can also be extended to functions invariant under a compact group of automorphisms of $G$ (the group of automorphisms of $G$ is given the compact-open topology).

In addition, the main results of this paper can be extended to locally compact groups. There $\mathscr{E}$ is replaced by the space of continuous functions, $\mathscr{D}$ the space of continuous functions of compact carrier, $\mathscr{D}^{\prime}$ the space of measures and $\mathscr{E}^{\prime}$ the space of measures of compact carrier.

\section{REFERENCES}

1. S. Banach, Théorie des operation linéaires, Warsaw, 1932.

2. C. Chevalley, Theory of Lie groups, Princeton, 1946.

3. J. Dieudonné, Recent developments in the theory of locally convex vector spaces, Bull. Amer. Math. Soc., 59 (1954), 495-512

4. __ and L. Schwartz, La dualité dans les espaces $(F)$ et $(L F)$, Annales de l'Institut Fourier de Grenoble, 1 (1949), 61-101.

5. L. Ehrenpreis, Theory of distributions for locally compact spaces, Columbia University Thesis (to appear in Memoirs Amer. Math. Soc.)

6. Analytic functions and the Fourier transform of distributions, I, Ann. of Math., 63 (1956), 129-159.

7. _- Analytic functions and the Fourier transform of distributions II, (to appear).

8. and F. I. Mautner, Some properties of the Fourier transform on semisimple Lie groups, (to appear).

9. F. I. Mautner, Unitary representations of locally compact groups I, Ann. of Math., 51 (1950), 1-25.

10. Unitary representations of locally compact groups II, Ann. of Math., 52, (1950), 528-556.

11. L. Pontrjagin, Topological groups, Princeton, 1939.

12. G. de Rham and K. Kodaira, Harmonic integrals, The Institute for Advanced Study, 1950 (mimeographed).

13. L. Schwartz, Théorie des distributions, vols. I and II, Paris, 1950-51.

14. A. Weil, L'Intégrations dans les groupes topologiques et ses applications, Paris, 1940.

The Institute for Advanced Study 



\section{PACIFIC JOURNAL OF MATHEMATICS}

\section{EDITORS}

H. L. Royden

Stanford University

Stanford, California

E. Hewits

University of Washington

Seattle 5 , Washington
R. P. Dilworth

California Institute of Technology Pasadena 4, California

E. G. Straus

University of California

Los Angeles 24, California

\section{ASSOCIATE EDITORS}
E. F. BECKENBACH
C. E. BURGESS
H. BUSEMANN
H. FEDERER

\author{
M. HALL \\ P. R. HALMOS \\ V. GANAPATHY IYER \\ R. D. JAMES
}

\author{
M. S. KNEBELMAN \\ I. NIVEN \\ T. G. OSTROM \\ M. M. SCHIFFER
}

\section{J. J. STOKER \\ G. SZEKERES \\ F. WOLF \\ K. YOSIDA}

\section{SUPPORTING INSTITUTIONS}

UNIVERSITY OF BRITISH COLUMBIA CALIFORNIA INSTITUTE OF TECHNOLOGY

UNIVERSITY OF CALIFORNIA

MONTANA STATE UNIVERSITY

UNIVERSITY OF NEVADA

OREGON STATE COLLEGE

UNIVERSITY OF OREGON

UNIVERSITY OF SOUTHERN CALIFORNIA
STANFORD UNIVERSITY

UNIVERSITY OF UTAH

WASHINGTON STATE COLLEGE

UNIVERSITY OF WASHINGTON

$*$ * * *

AMERICAN MATHEMATICAL SOCIETY

CALIFORNIA RESEARCH CORPORATION HUGHES AIRCRAFT COMPANY

Mathematical papers intended for publication in the Pacific Journal of Mathematics should be typewritten (double spaced), and the author should keep a complete copy. Manuscripts may be sent to any of the editors. Manuscripts intended for the outgoing editors should be sent to their successors. All other communications to the editors should be addressed to the managing editor, E. G. Straus at the University of California, Los Angeles 24, California.

50 reprints of each article are furnished free of charge; additional copies may be obtained at cost in multiples of 50 .

The Pacific Journal of Mathematics is published quarterly, in March, June, September, and December. The price per volume (4 numbers) is $\$ 12.00$; single issues, $\$ 3.50$. Back numbers are available. Special price to individual faculty members of supporting institutions and to individual members of the American Mathematical Society: $\$ 4.00$ per volume; single issues, $\$ 1.25$.

Subscriptions, orders for back numbers, and changes of address should be sent to Pacific Journal of Mathematics, 2120 Oxford Street, Berkeley 4, California.

Printed at Kokusai Bunken Insatsusha (International Academic Printing Co., Ltd.), No. 10, 1-chome, Fujimi-cho, Chiyoda-ku, Tokyo, Japan.

\section{PUBLISHED BY PACIFIC JOURNAL OF MATHEMATICS, A NON-PROFIT CORPORATION}

The Supporting Institutions listed above contribute to the cost of publication of this Journal, but they are not owners or publishers and have no responsibility for its content or policies. 


\section{Pacific Journal of Mathematics}

\section{Vol. 6, No. 4}

1956

Seymour Ginsburg, On mappings from the family of well ordered subsets of

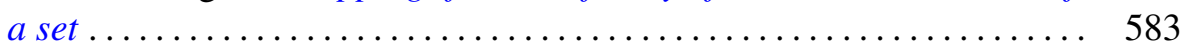

Leon Ehrenpreis, Some properties of distributions on Lie groups ......... 591

Marion K. Fort, Jr., A geometric problem of Sherman Stein ............. 607

Paul R. Garabedian, Calculation of axially symmetric cavities and jets . . . . 611

Walter Mossman Gilbert, Completely monotonic functions on cones ...... 685

William L. Hart and T. S. Motzkin, A composite Newton-Raphson gradient method for the solution of systems of equations ................. 691

C. W. Mendel and I. A. Barnett, A functional independence theorem for square matrices ................................. 709

Howard Ashley Osborn, The problem of continuous programs .......... 721

William T. Reid, Oscillation criteria for linear differential systems with complex coefficients ............................. 733

Irma Reiner, On the two-adic density of representations by quadratic

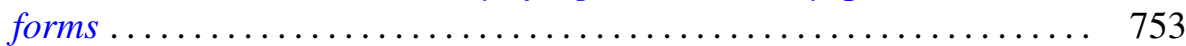

Shoichiro Sakai, A characterization of $W^{*}$-algebras .............. 763

Robert Steinberg, Note on a theorem of Hadwiger................. 775

$\mathrm{J}$. Eldon Whitesitt, Construction of the lattice of complemented ideals within

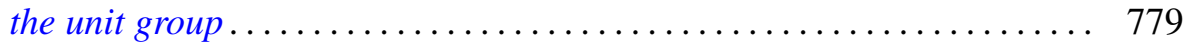

Paul Civin, Correction to "Some ergodic theorems involving two operators"... 